\title{
Nutrition and lysosomal activity. The effect of dietary cod-liver oil on the distribution of polyunsaturated fatty acids in the kidney lysosomes of rats receiving deficient or adequate intakes of vitamin $E$
}

\author{
By M. IQBAL,* J. T. DINGLE AND T. MOORE \\ Strangeways Research Laboratory, Cambridge \\ AND I. M. SHARMAN \\ Dunn Nutritional Laboratory, University of Cambridge \\ and Medical Research Council
}

(Received 8 April 1968-Accepted 2 August 1968)

I. In an attempt to explain the antagonism between polyunsaturated fatty acids (PUFA) and vitamin $E$ in the promotion of post-mortem autolysis and increased lysosomal fragility in the kidney of rats, studies were made by gas chromatography of the incorporation of PUFA into the lysosomes.

2. Kidneys were taken from rats that had received various diets, which differed in their fat components and which were with or without vitamin $E$. Since the inclusion of cod-liver oil in the diet reduces the period of dietary preparation necessary for rapid kidney autolysis, the effect of this oil on the PUFA distribution in the lysosomes was specially studied.

3. In purified preparations of kidney lysosomes from rats that had received substantial amounts of cod-liver oil for several weeks, $\mathrm{C} 20: 5$ acid was incorporated mainly at the expense of $\mathrm{C}_{1} 8: 2$ (linoleic) and $\mathrm{C}_{20: 4}$ (arachidonic) acids. In less purified lysosomal fractions the incorporation of $\mathrm{C}_{20}: 5$ and $\mathrm{C}_{22}: 6$ acids and the corresponding reductions in linoleic and arachidonic were well advanced after Io days, but were not maximal until about 30 days. The same changes took place in the reverse direction, with about the same rapidity, when rats that had previously been given cod-liver oil were changed to a diet containing lard.

4. The percentage of PUFA in the kidney lysosomes of rats not dosed with vitamin $E$ was not significantly different from that of rats given adequate doses.

5. Thus the increased tendency to kidney autolysis, and the reduced stability of the lysosomes, caused by the feeding of cod-liver oil were associated with the partial replacement of linoleic acid and of the endogenous arachidonic acid by an acid, usually foreign to the rat, which is even more unsaturated. Since vitamin E did not prevent the entry of this acid into the lysosomes its potency in retarding autolysis and stabilizing the lysosomes must be exerted at some point subsequent to the incorporation of PUFA.

6. Since change in the percentage of lysosomal PUFA in response to dietary changes is slow, this suggests that the lysosomal lipids escape usage in general metabolism as an immediate source of calories and that they have a half-life of at least I $_{5}$ days. Individual fatty acids, however, may differ in their half-lives.

Moore, Sharman, Stanton \& Dingle (1967) have shown that the action of vitamin E in retarding post-mortem autolysis in the kidney cortex of rats (Emmel, 1956; Moore, Sharman \& Symonds, 1958) is linked with its power to stabilize the kidney lysosomes. The presence in the diet of polyunsaturated fatty acids (PUFA) works in the opposite direction. Thus Moore et al. (1967) demonstrated that the inclusion in the diet of cod-liver oil, rich in $\mathrm{C}_{20}: 5$ and $\mathrm{C}_{22}: 6$ acids, reduced the period of dietary prepara-

* Present address: Department of Oral Pathology, College of Dentistry, University of Illinois at Medical Center, Chicago, Illinois, USA. 
tion necessary to accelerate autolysis and also rendered the kidney lysosomes less stable.

As a working hypothesis, to explain the antagonism between vitamin $\mathrm{E}$ and PUFA, we might assume that the inclusion of PUFA in the lysosomes, abnormal either in amount or in quality, might reduce the stability of the lysosomal membrane. Vitamin $E$ might act either by controlling the incorporation of PUFA into the membranes, or by decreasing the liability of PUFA to become oxidized after incorporation.

To test this hypothesis the influence of dietary PUFA and vitamin $\mathrm{E}$ on the relative amounts of fatty acids present in the kidney lysosomes was investigated. Because of the convenience of cod-liver oil for producing the effects under investigation this oil was given in most of our experiments; for purposes of comparison, some rats received lard instead of cod-liver oil, or diets low in fat.

\section{EXPERIMENTAL AND RESULTS}

\section{Materials and methods}

Rats. Piebald rats of both sexes were used but usually in each experiment and always in each group of rats, all the animals were of the same sex. Most of them were taken as weanlings, from mothers fed on the cubed diet $4 \mathrm{I}$ B of Oxoid Limited, and were given dietary preparations as detailed later.

Diets. The experimental diets and the preparation of cod-liver oil low in vitamin A, so as to avoid danger of hypervitaminosis, were as reported by Moore et al. (1967). Our 'cod-liver oil (CLO) diet' contained 10\% of the oil, and the 'lard diet' 10\% of lard.

Preparation of lysosomal fractions. For most of our work a crude lysosomal fraction was isolated by a short process which had the advantage that enough material could be obtained without using large groups of rats. This method was used, for example, to find the rate of appearance of PUFA from cod-liver oil in the kidney lysosomes after the oil had been included in the diet, and the rate of disappearance after the withdrawal of the oil. It seemed important, however, to establish the presence of PUFA from cod-liver oil in the purest obtainable lysosomal fraction. In special experiments, therefore, kidneys from large groups of rats were pooled for the preparation of lysosomes, by a lengthier process (see below).

Crude lysosomal fractions. Two animals from each group were killed by cervical dislocation and the kidneys were removed and immediately placed in ice-cold $0.25 \mathrm{M}$ sucrose. One quarter of a kidney from each rat was taken for histological examination and the rest were cut up with scissors. The pulp was homogenized in 9 vol. $(w / v)$ of ice-cold $0.25 \mathrm{M}$-sucrose in a Potter-Elvehjem type homogenizer, consisting of a glass tube and teflon pestle. The homogenate was spun at $600 \times g$ for $5 \mathrm{~min}$ in a (MSE refrigerated) centrifuge at $4^{\circ}$. The pellet, consisting of nuclei and unbroken cells was discarded and the supernatant fraction was spun at $16000 \times g$ for $20 \mathrm{~min}$. The pellet, consisting of mitochondria and lysosomes, was removed and either used immediately or stored at $-20^{\circ}$ under nitrogen for not more than 2 days.

Pure lysosomal fractions. Twelve rats from each group were killed and the blood 
was washed from the vascular system of the kidneys by perfusion of cold $0.3 \mathrm{M}$-sucrose solution buffered at $\mathrm{pH} 7.0$ with I mv-EDTA. The kidneys were removed, blotted on filter-paper and weighed, and homogenized in $0.44 \mathrm{M}$-sucrose solution $\mathrm{pH} 7$ to give a $6 \%$ homogenate. A lysosomal fraction of high purity, as indicated by microscopic examination, was obtained by a slight modification of Maunsbach's (I966) method.

Extraction of lipids from diet. Lipids present in the diet were usually extracted by a modification of the method of Folch, Lees \& Stanley (1957). The diet was first treated with 20 vol. of chloroform-methanol-water $(38: 19: 3, v / v)$ and the extract was removed by filtration. The residue was re-extracted with chloroform-methanol $(2: \mathrm{I}, \mathrm{v} / \mathrm{v})$ by allowing it to stand overnight at room temperature with a little water. The extracts were combined and washed with 0.2 vol. of $0.29 \% \mathrm{NaCl}$ solution. In some instances a simple extraction with hot ethanol was found more convenient. Application of both methods to the same material gave similar results.

Extraction of tissues. Total lipid extracts of crude lysosomes and pure granules were prepared as described by Folch $e$ al. (1957). The lipid extract was deproteinized by evaporating the extracts in chloroform-methanol-water $(64: 32: 4, \mathrm{v} / \mathrm{v})$ to dryness, extracting with $\mathrm{CHCl}_{3}$ and filtering to remove the insoluble proteins.

Gas-liquid chromatography. The extracted lipids were saponified in $90 \%$ ethanolic $\mathrm{KOH}\left(\mathrm{I} \mathrm{ml} \mathrm{33 \%} \mathrm{KOH} \mathrm{to} \mathrm{which} \mathrm{were} \mathrm{added} 9 \mathrm{ml}\right.$ ethanol solution) at $55^{\circ}$ for $20 \mathrm{~min}$ and free fatty acids were liberated by the addition of $50 \%(\mathrm{v} / \mathrm{v}) \mathrm{HCl}$ until the solution gave a slight acid reaction. Fatty acids were usually extracted by hexane, and methyl esters were formed by reaction with freshly prepared diazomethane in ether until the solution remained permanently yellow. After I $5 \mathrm{~min}$, the excess of diazomethane was evaporated with a stream of nitrogen and the methyl esters were purified by thinlayer chromatography on silica gel $\mathrm{G}$. The plates were developed with hexane: ether $(95: 5)$ and the methyl esters eluted with chloroform.

Methyl esters were separated on a Panchromatograph fitted with argon detector (Pye Limited, Cambridge) at $185^{\circ}$. The column was a glass tube packed with polythene-glycol-adipate coated on graded celite grains. Four $\mu 1$ of the sample were analysed, with the argon flow rate at $60 \mathrm{ml} / \mathrm{min}$, the detector voltage at $550 \mathrm{~V}$ and the backing off current at $10^{-8} \mathrm{~A}$. The chromatographs were analysed by triangulation and the proportion of individual fatty acids was expressed as percentages of the total. Pure fatty acids (Sigma Chemical Company) were used as standards. Percentages have been based on the total of the main acids as listed in each table; the possibility that small amounts of other acids may have been present is not excluded.

Measurement of lysosomal instability. In a procedure described in detail by Dingle, Sharman \& Moore (1966) the kidney tissues were homogenized and submitted to ultracentrifugation as for the preparation of crude lysosomes. 'Free' acid protease activity $(F)$ was then measured in the supernatant fraction, and 'bound' activity $(B)$ in the lysosomal pellet after its treatment with detergent. Lysosomal fragility was assessed as $F /(F+B) \times 100$, on the assumptions that all the proteolytic activity in the supernatant fraction was derived from broken lysosomes, and that the percentage of lysosomes broken during the homogenization procedure depended on their fragility.

Kidney autolysis. Autolysis was assessed by preparing paraffin sections of kidneys 
that had been fixed $3 \mathrm{~h}$ after the death of the rat, staining them with haematoxylin and eosin, and observing the loss of structure in the cortical tubules (Moore et al. I958).

\section{Experimental procedure and results}

Fatty acids in diets and dietary components. Values for fatty acids in diet $4 \mathrm{I} \mathrm{B}$, bread, cod-liver oil, lard and dried yeast, as found by gas chromatography, are given in Table I.

Expt I: pure and crude lysosomal fractions. One preparation of pure lysosomes was made from the combined kidneys of a large group of rats that had been fed for 3 weeks after weaning on diet $4 \mathrm{I} \mathrm{B}$, the same food as had been given to their mothers. Two other preparations were made from the kidneys of groups that had been fed for the

Table I. Distribution of fatty acids, as percentages of the total, in fat extracted from diet $4 \mathrm{I} B$, bread and dried yeast, and in the dietary fats, lard and cod-liver oil

\begin{tabular}{|c|c|c|c|c|c|}
\hline Fatty acid & Diet $4 \mathrm{r} B$ & Bread & Dried yeast & Lard & Cod-liver oi \\
\hline$C_{10}$ & - & - & $4 \cdot 6$ & $\cdots$ & 0.9 \\
\hline $\mathrm{C}_{12}$ & - & - & 5.0 & - & - \\
\hline $\mathrm{C}_{\mathrm{I} 4}$ & 0.7 & 0.6 & $2 \cdot 0$ & I.5 & $2 \cdot 5$ \\
\hline $\mathrm{C}_{14: \mathrm{I}}$ & - & - & I. 5 & - & - \\
\hline C 16 & 16.7 & $20 \cdot 6$ & 33.4 & $30 \cdot 3$ & $12 \cdot 3$ \\
\hline$C_{16: 1}$ & $\mathrm{I}-6$ & $2 \cdot I$ & 33.0 & 3.0 & 8.4 \\
\hline $\mathrm{C}_{16: 2}$ & - & - & - & - & $1 \cdot 3$ \\
\hline $\mathrm{C}_{18} 8$ & $x \cdot 3$ & $6 \cdot 5$ & 74 & $17 \cdot 3$ & $3 \cdot 9$ \\
\hline$C_{18: 1}$ & $29 \cdot I$ & $26 \cdot 8$ & 10.9 & $41 \cdot 8$ & $27 \cdot 5$ \\
\hline $\mathrm{C}_{18: 2}$ & $47 \cdot 8$ & $36 \cdot 8$ & $2 \cdot 2$ & $6 \cdot I$ & 2.9 \\
\hline$C_{18: 3}$ & $1 \cdot 9$ & $2 \cdot I$ & - & - & 1.5 \\
\hline $\mathrm{C}_{18: 4}$ & - & - & 一 & - & $2 \cdot 9$ \\
\hline $\mathrm{C}_{20: \mathrm{I}}$ & 0.9 & 0.9 & 一 & - & 12.5 \\
\hline $\mathrm{C}_{20: 4}$ & - & - & - & - & $I \cdot 5$ \\
\hline C 20:5 & - & - & - & - & 8.8 \\
\hline$C_{22: 1}$ & - & $3.6 *$ & - & - & $5 \cdot 2$ \\
\hline$C_{22: 6}$ & - & - & - & - & 7.9 \\
\hline
\end{tabular}

* Kindly identified by Dr N. W. R. Daniels, of the Technological Research Station of Spillers Limited, by comparing gas chromatography traces before and after bromination and hydrogenation of the esters. Presumably it came from the small percentage of fat, of variable origin, which is always added to the dough during bread making.

same time on a diet containg $10 \%$ cod-liver oil, with supplements of $3 \mathrm{mg}$ DL- $\alpha$ tocopheryl acetate twice weekly. Crude lysosomal fractions were prepared from three smaller groups of young rats given the diet $4 \mathrm{I}$ B and from two other groups that received the diet containing cod-liver oil. (The last-mentioned groups formed part of Expt 2, described below.) In Table 2, fatty acid distributions in the pure and crude lysosome fractions are compared. In the kidneys of the rats given cod-liver oil substantial amounts of $\mathrm{C} 20: 5$ were incorporated into the pure lysosomes. Both $\mathrm{C} 20: 5$ and $\mathrm{C} \mathrm{22:6}$ acids were found in the crude lysosome fractions.

Expts 2 and 3: rate of incorporation of $C 20: 5$ and $\mathrm{C} \mathrm{22}_{2} 6$ fatty acids into crude lysosomal fractions in response to the feeding of cod-liver oil, and their dispersal after the withdrawal of cod-liver oil. The incorporation of acids was studied in Expt 2 and their 
dispersal in Expt 3. These experiments were made at different times and on rats of different sexes, but were so designed that their results could be combined.

To provide a base-line for studies on the effect of feeding cod-liver oil, the mean findings on the three groups of rats fed on diet $4 \mathrm{IB}$, mentioned above, were used. In Expt 2, two large groups of male weanling rats were given the diet containing codliver oil, with or without supplements of DL- $\alpha$-tocopheryl acetate. Two or three rats of each group were killed after 10, 2I, 31,42 and 52 days, and the fatty acid distributions in crude kidney lysosomal fractions were studied. In Expt 3, weanling female rats were first fed on the diet containing cod-liver oil, with or without tocopherol, for

Table 2. Percentage fatty acid distribution in pure and impure lysosomal fractions from the kidneys of rats fed upon a stock diet $4 \mathrm{I} B$, or on a diet containing $10 \%$ of cod-liver oil

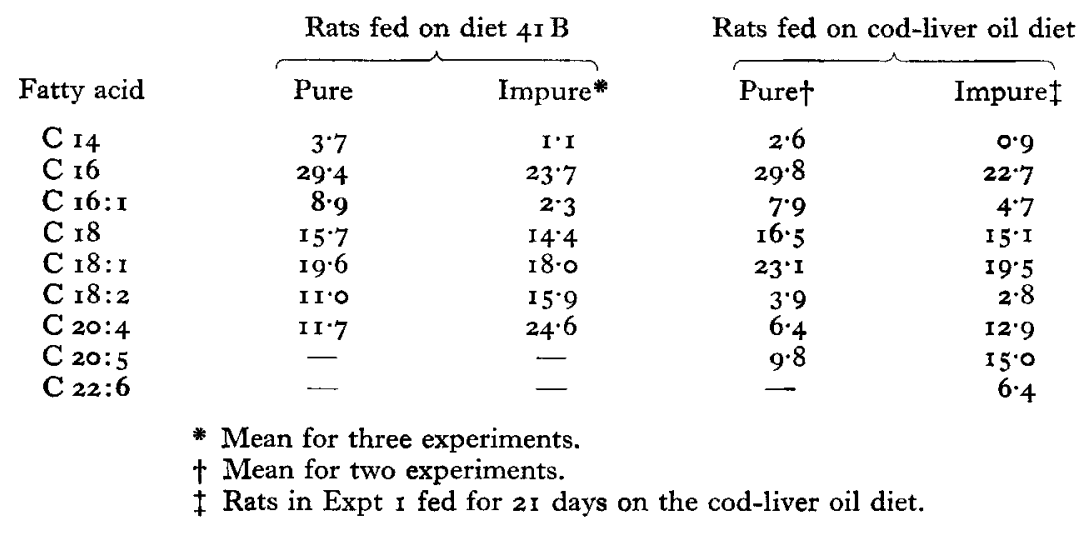

3 days. Two or three rats from each group were then killed for examination of the fatty acids of their kidney lysosomes. The remaining rats were changed to a diet in which lard was substituted for the cod-liver oil and were killed after a further 3, 7, I4 or 28 days. Even 28 days was insufficient to allow the changes in the fatty acid composition in the lysosomal fractions to reach completion. An additional experiment was therefore made on a group of rats that received no cod-liver oil but were fed for $3 \mathrm{I}$ days after weaning on the diet containing lard.

In the many estimations of individual fatty acids made in these experiments differences were occasionally found between groups dosed and undosed with vitamin $E$ that were greater than would have been expected for duplicate assays on the same material. These differences, however, were inconsistent in direction. To test this point further, the fatty acid percentages found in Expt 2 for dosed and undosed animals were averaged for a combination of days $3^{1}, 4^{2}$ and $5^{2}$, the previous results being omitted because the incorporation of $\mathrm{C} 20: 5$ and $\mathrm{C} 22: 6$ acids had not reached a maximum. The average percentages, given in Table 3 , failed to show that vitamin $\mathrm{E}$ had any influence on the proportions of individual acids incorporated.

For this reason, and to reduce the effect of minor experimental variations, the results in Expts 2 and 3 for groups dosed or undosed with vitamin $E$ were averaged for each day on which rats were killed. The chief changes caused by the feeding of cod-liver 
Table 3. Distribution of fatty acids in impure lysosomal fractions from rats fed upon a diet containing cod-liver oil, with or without dosing with vitamin $E$

(Each result is the combined mean for groups of rats killed after receiving the diet for $3 \mathbf{I}$, 42 or 52 days)

\begin{tabular}{|c|c|c|}
\hline Fatty acid & $\begin{array}{l}\text { Without } \\
\text { vitamin E }\end{array}$ & $\begin{array}{c}\text { With } \\
\text { vitamin }\end{array}$ \\
\hline $\mathrm{C}_{\mathrm{I} 4}$ & $1 \cdot 0$ & $I \cdot I$ \\
\hline$C=6$ & 19.2 & $20 \cdot 8$ \\
\hline$C_{16: 1}$ & 3.9 & $4 \cdot 4$ \\
\hline C I 8 & $17 \cdot 2$ & 15.8 \\
\hline C I8: I & I 5.9 & $16 \cdot 6$ \\
\hline $\mathrm{C} 18: 2$ & $3 \cdot 1$ & $2 \cdot 1$ \\
\hline C $20: 4$ & 14.6 & $x_{4} \cdot 6$ \\
\hline $\mathrm{C}_{20: 5}$ & I 7.5 & 17.0 \\
\hline C 22:6 & $7 \cdot 6$ & $7 \cdot 6$ \\
\hline
\end{tabular}

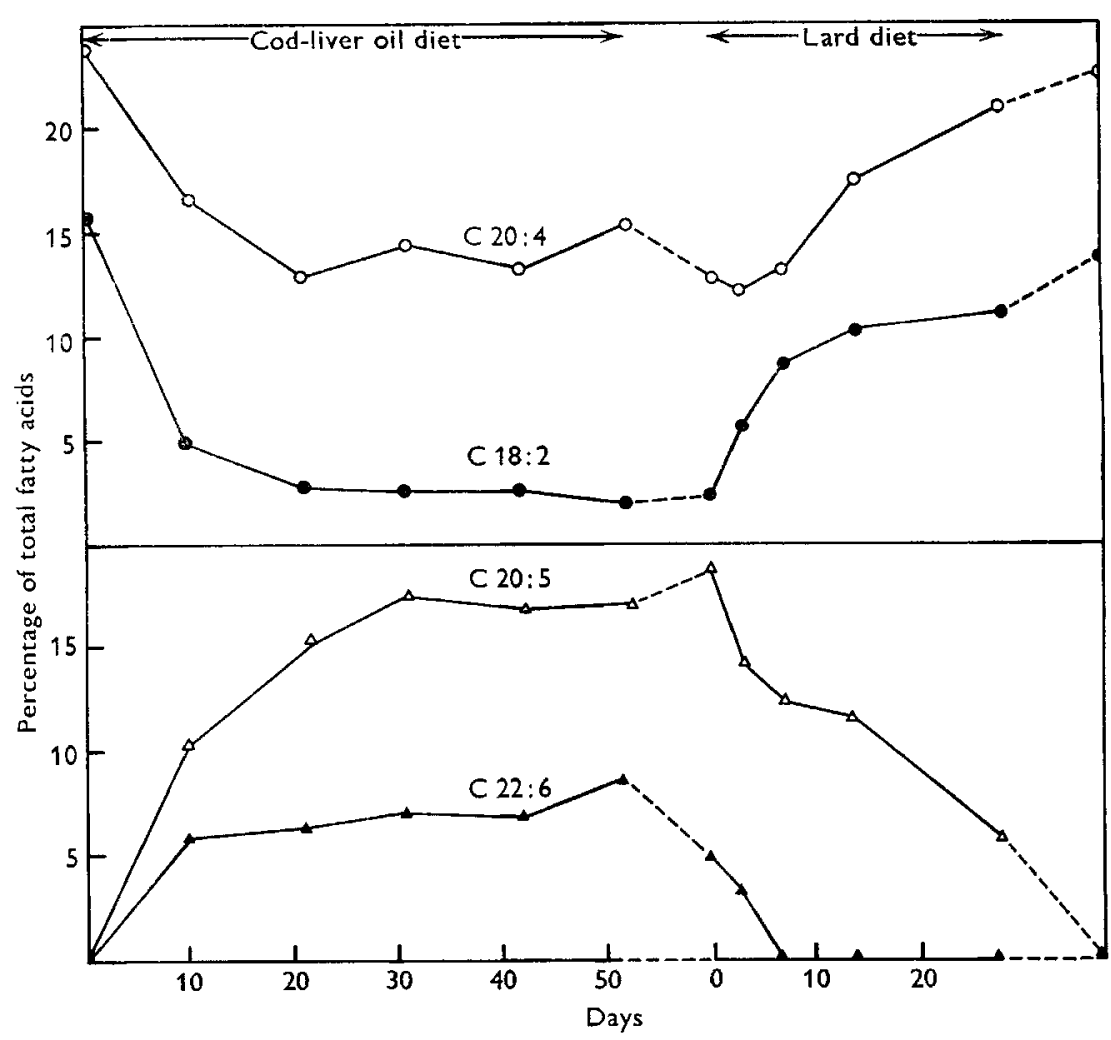

Fig. I. Incorporation into the crude kidney lysosomal fraction of $\mathrm{C} 20: 5$ and $\mathrm{C} z 2: 6$ acids derived from the dietary cod-liver oil, and the corresponding displacement of $C$ I8:2 and $\mathrm{C} 20: 4$ acids. The readings at day 0 , on the left, refer to rats reared on the stock diet, 4 I $B$. At this point a diet containing $10 \%$ of cod-liver oil was substituted and continued until day 52 of the experiment. At the point $o$, to the right of the diagram, rats which had previously received a diet containing cod-liver oil for $3 \mathbf{I}$ days were transferred to a diet containing lard. The final points on the extreme right side refer to rats which received a diet containing lard, without first receiving the cod-liver oil diet. Broken lines in the graphs or time axis indicate discontinuity in time and in the arrangement of the experiment. Each point, except at the two extremes, is the mean for rats dosed or undosed with vitamin $\mathrm{E}$. 
oil, already mentioned above, were the introduction of $\mathrm{C} 20: 5$ and $\mathrm{C} 22: 6$ acids, which are normally foreign to the rat. The inclusion of these acids was mainly at the

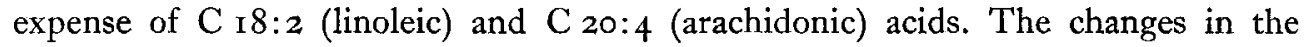
proportions of these four acids with time are shown in Fig. $I$.

The influence of vitamin $E$ on the effects of cod-liver oil. In Expt 2, observations were made not only on the fatty acid composition in the lysosomes, but on the growth rates of the rats, the colour of their body fat, post-mortem autolysis of their kidneys and the fragility of their kidney lysosomes. As expected, cod-liver oil greatly retarded the growth rate, and even caused the death of a few of the animals. Simultaneous

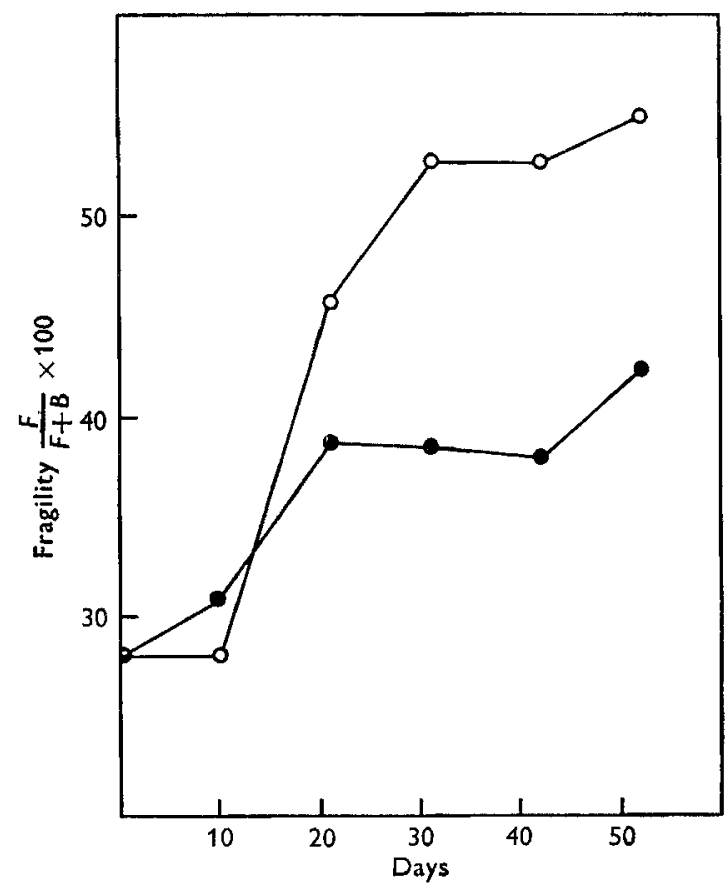

Fig. 2. Expt 2. Effect of supplements of vitamin $E$ on the fragility of crude kidney lysosome fractions from rats fed for increasing periods on a diet containing cod-liver oil. 0 , undosed rats; $\bullet$, rats dosed with $\alpha$-tocopheryl acetate.

dosing with $\alpha$-tocopheryl acetate, however, counteracted the toxic effects of cod-liver oil. Towards the end of the experiment the dosed animals were usually about roo $g$ heavier than the undosed, with mean weights of $308 \mathrm{~g}$ and $205 \mathrm{~g}$ respectively in pairs of rats killed after 52 days. The vitamin also prevented brown discoloration of the body fat. In confirmation of previous findings (Moore et al. I967), cod-liver oil accelerated the tendency to kidney autolysis, as observed histologically, and increased the fragility of the kidney lysosomes, as measured by the release of acid protease. Dosing with $\alpha$-tocopherol prevented abnormal autolysis and at least partially protected the kidney lysosomes against increased fragility (Fig. 2).

Rats fed on bread. In a separate investigation, rats were weaned and kept for 9 months on a diet of white bread, supplemented only with adequate doses of vita- 
min $A$, and with or without vitamin E. It seemed of interest to examine the kidney lysosomes from these animals in view of the very low fat contents of their diet. The percentages of fatty acids in the kidney lysosomes, averaged for rats dosed and undosed

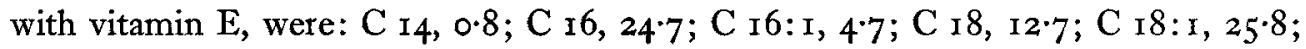
$\mathrm{C}_{18} 8: 2,9 \cdot 5 ; \mathrm{C}_{20: 4}, 21 \cdot 8$.

\section{DISCUSSION}

Dietary PUFA and kidney abnormalities. Emmel (1956) and Moore et al. (1958) reported that diets low in PUFA, either through the absence of fat or the inclusion of fats lacking in PUFA, do not give rise to rapid autolysis even when the diet is deficient in vitamin E. Such diets, however, cause other kidney injuries, manifested by haematuria (Burr \& Burr, 1930). The kidney therefore requires an adequate intake of PUFA, supplemented by vitamin $\mathrm{E}$.

The incorporation and displacement of lysosomal PUFA. The results of Expts $\mathrm{I}$ and 2 indicate that the inclusion of cod-liver oil in the diet, which increases the tendency to rapid post-mortem autolysis in the kidney, also causes the incorporation of foreign $\mathrm{C} 20: 5$ acid in the lysosomes of this organ. The appearance of $\mathrm{C} \mathrm{20:5}$ acid is accompanied by decreases in the $\mathrm{C} I 8: 2$ and $\mathrm{C} 20: 4$ acids normally present in the lysosomes.

Since our estimations were made on the percentages of acids rather than on the absolute amounts, the inclusion of any new acids must inevitably have reduced the percentages of those originally present. Reductions from this cause would have been about $20 \%$ if spread evenly over all the original acids. Our findings, however, were that linoleic acid was reduced on an average by $83 \%$, and arachidonic by $39 \%$, as compared with reductions of only $15 \%$ for palmitic acid and $8 \%$ for oleic acid.

Clearly $\mathrm{C}$ 20:5 acid was selectively absorbed from the dietary cod-liver oil into the kidney lysosomes. Thus the percentage of this acid in the lysosomes, after the prolonged feeding with cod-liver oil, was twice that found in the dietary fat. Moreover $\mathrm{C} 20: 5$ acid was incorporated in preference to $\mathrm{C}_{22: 6}$. Although these acids were present in about equal amounts in the cod-liver oil much more $C_{20: 5}$ than $C_{22}: 6$ was always present in crude lysosomal preparations, while in pure lysosomes no $\mathrm{C} 22: 6$ was found. On the withdrawal of cod-liver oil from the diet, $\mathrm{C}$ 22:6 disappeared from crude kidney lysosomes more rapidly than did C 20:5 (Expt 3).

The PUFA requirements of lysosomes, and doubtless of other cell components, appear to be specific, with the endogenous $\mathrm{C}_{20: 4}$ acid, arachidonic acid, playing a key role. This was borne out in our experiments on rats fed upon bread, or upon a diet containing lard. These animals received virtually no arachidonic acid in their food, but it was present as $22.3 \%$ and $23 \%$ respectively of the fatty acids of their crude kidney lysosomes. Presumably it was synthesized from linoleic acid contained in the diet. When the supply of arachidonic acid to the cells is deficient, on account of dietary lack of the essential linoleic acid, the cells must find a substitute. During cod-liver oil feeding, $\mathrm{C}_{20: 5}$ acid is incorporated presumably because it is the nearest substitute. During the incorporation into membrane lipids, a dynamic system of bio-chromatography may perhaps be visualized, with closely similar acids, such as $\mathrm{C} 20: 4$ and $\mathrm{C} 20: 5$, competing for the same site. 
PUFA, vitamin $E$ and kidney autolysis. The accelerated autolysis caused by the feeding of cod-liver oil, as observed by Moore et al. (1967) and confirmed in Expt 2 (Fig. 2), is therefore associated with an increase in the degree of unsaturation in the lysosomal PUFA, which presumably become more sensitive to oxidation. We must not conclude, however, that the lysosomes are exceptional among organelles for their high content or specific demands for PUFA. Comparison of our results on pure and crude lysosomal fractions indicates that the percentage of the $\mathrm{C} 20: 5$ acid was greater in the less pure fractions. C 22:6 acid was present in the impure, but absent from the pure, fractions. The reason for the particular liability of the kidney cortex to autolysis remains obscure, as also does the role of vitamin $\mathrm{E}$ in preventing autolysis. Our findings, in Expts 2 and 3, that dosing with vitamin $\mathrm{E}$ failed to influence the PUFA content of the lysosomes excluded the possibility that it acts by controlling the incorporation of PUFA into these organelles. Probably a complex mechanism is involved, which may include effects of PUFA and vitamin $E$ on oxidative changes in the mitochondria, which can in turn affect the lysosomes.

The life period of the lysosomal PUFA. Our Expt 2, on the rate of incorporation of C 20:5 into kidney lysosome fractions, and Expt 3, on the rate of disappearance of this acid, indicate that the adjustment of the lysosomal lipids under the influence of dietary fat is a slow process. Thus under our experimental conditions about 30 days were required for the incorporation of this acid to reach a maximum. On the removal of cod-liver oil from the diet in Expt 3 the half life of the $\mathrm{C} 20: 5$ acid appeared to be about ${ }_{1} 5$ days. These slow responses to dietary changes suggest that PUFA have a structural role in lysosomes, and presumably in other organisms, which goes beyond their role as a non-specific source of energy.

Our thanks are tendered to Dame Honor Fell, FRS and Dr E. Kodicek for valuable criticism and to Miss Bridget Bunter and Mr Peter Martin for skilled technical help. J.T.D. is a member of the External Staff of the Medical Research Council and T.M. receives a part-time grant.

\section{REFERENCES}

Burr, G. O. \& Burr, M. M. (I930). 7. biol. Chem. 86, 587.

Dingle, J. T., Sharman, I. M. \& Moore, 'T. (1966). Biochem. F. 98, 476.

Emmel, V. M. (1956). Anat. Rec. 124, 399.

Folch, J., Lees, M. \& Stanley, G. H. S. (1957). F. biol. Chem. 226, 497.

Maunsbach, A. B. (1966). F. Ultrastruc. Res. 16, I3.

Moore, T., Sharman, I. M., Stanton, M. G. \& Dingle, J. T. (1967). Biochem. F. ro3, 923.

Moore, T., Sharman, I. M. \& Symonds, K. R. (1958). F. Nutr. 65, I 83 . 